\title{
Review Article \\ Effects of Uric Acid on Diabetes Mellitus and Its Chronic Complications
}

\author{
Qing Xiong $\mathbb{D}^{1,2}$ Jie Liu $\mathbb{D D}^{1}{ }^{1}$ and Yancheng $X u \mathbb{D}^{1}$ \\ ${ }^{1}$ Department of Endocrinology, Zhongnan Hospital of Wuhan University, Wuhan, Hubei 430071, China \\ ${ }^{2}$ Department of Endocrinology, Affiliated Haikou Hospital of Xiangya Medical College, Central South University, Haikou, \\ Hainan 570208, China \\ Correspondence should be addressed to Yancheng Xu; xjl100901@whu.edu.cn
}

Received 28 March 2019; Revised 26 May 2019; Accepted 6 September 2019; Published 10 October 2019

Guest Editor: Arcidiacono Biagio

Copyright (C) 2019 Qing Xiong et al. This is an open access article distributed under the Creative Commons Attribution License, which permits unrestricted use, distribution, and reproduction in any medium, provided the original work is properly cited.

With the deepening of the researches on uric acid, especially in the study of metabolic diseases, uric acid has been found to be closely related to obesity, metabolic syndrome, nonalcoholic fatty liver disease, diabetes, and other metabolic diseases. Uric acid causes a series of pathophysiological changes through inflammation, oxidative stress, vascular endothelial injury, and so on and thus subsequently promotes the occurrence and development of diseases. This review confirmed the positive correlation between uric acid and diabetes mellitus and its chronic complications through the pathogenesis and clinical studies aspects.

\section{Introduction}

In recent years, human intake of foods such as those with the umami flavor (rich in purines), high added sugar (sucrose), and high fructose corn syrup have increased dramatically [1]. Fructose is the main component of added sugar. Unlike other sugars, fructose can cause mitochondrial oxidative stress $[2,3]$ and inhibits AMPK [4], and the subsequent intracellular ATP depletion [5] and nucleotide turnover lead to a significant increase in serum uric acid [6]. In addition to causing gout, many studies have shown that hyperuricemia is also closely related to cardiovascular diseases, metabolic syndrome, insulin resistance, and diabetes $[7,8]$. However, its function is a matter of debate [9]. Here, we reviewed the effects of hyperuricemia on diabetes and its complications and concluded that high levels of uric acid is closely related to diabetes and its chronic complications.

1.1. Uric Acid Formation. In the human body, uric acid is the ultimate product of purine metabolism (Figure 1 [10]). It is generated in the liver. Purine nucleotides decompose to hypoxanthine and guanine, some of which can be recycled and phosphorylated into hypoxanthine nucleotides, while the remaining part is metabolized by xanthine dehydrogenase/ oxidase $(\mathrm{XDH} / \mathrm{XO})$ enzymatic reaction to the terminal product uric acid. $\mathrm{XDH} / \mathrm{XO}$ is mainly expressed in the parenchymal cells of the liver and small intestine. $\mathrm{XDH}$ has low reactivity and can be converted to XO. Uric acid production primarily depends on the amount of substrate and the activity of $\mathrm{XO}$ [11]. In the end, $\mathrm{XDH} / \mathrm{XO}$ promotes the final steps in purine metabolism which convert hypoxanthine to xanthine and xanthine to UA [11]. The kidney also plays an important role in the regulation of blood uric acid levels. The circulating uric acid is easily filtered from the glomeruli into the renal tubule. About $90 \%$ of filtered UA is reabsorbed by the middle of the proximal convoluted tubule mainly by urate transporter 1 (URAT1) and glucose transporter 9 (GLUT9) [12], and the remaining excreted $10 \%$ is responsible for $60-70 \%$ of total body uric acid excretion $[13,14]$. A small amount of uric acid secreted in the intestine is responsible for $30-40 \%$ [14]. The production and excretion rate of uric acid is relatively constant in healthy people. Changes in the uric acid content in body fluids can reflect the state of metabolism, immunity, and other functions of the human body. If the body produces too much uric acid or the excretion mechanism is degraded, the body will retain excessive uric acid. Hyperuricemia was defined as the circulating uric acid levels of more than $5.7 \mathrm{mg} / \mathrm{dl}$ for women and $7.0 \mathrm{mg} / \mathrm{dl}$ for men [15]. When the blood uric acid concentration exceeds the norm, the human body fluid 


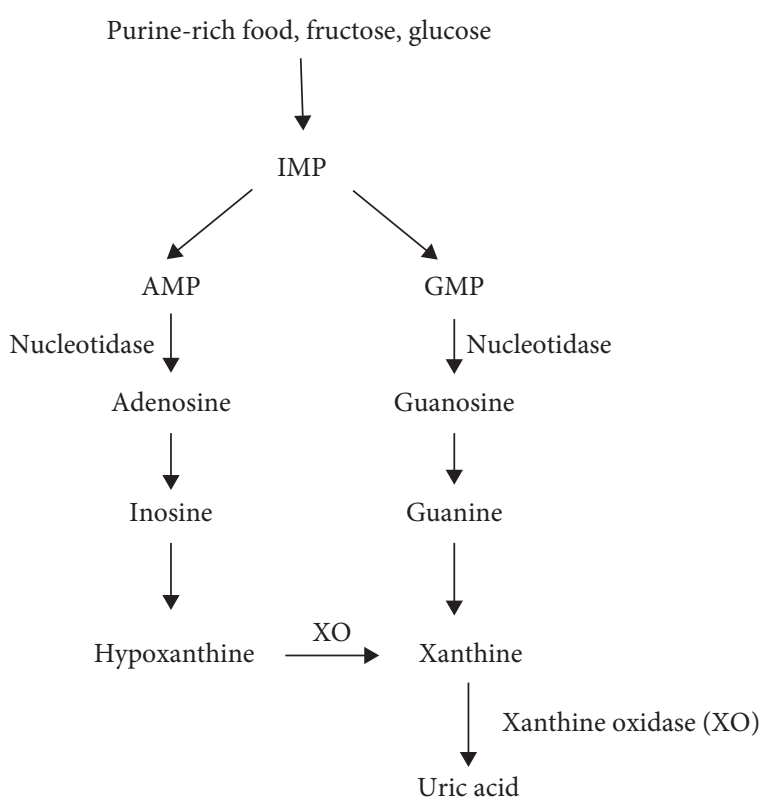

FIgURE 1: Process of purine metabolism in humans.

becomes acidic, which affects the normal function of the human cells, subsequently leading to metabolic disease in the long term [16-18].

\section{Pathological Mechanism of Uric Acid on Diabetes and Its Chronic Complications}

2.1. Uric Acid and Diabetes. At present, many studies have shown that the relevant pathological mechanisms include some aspects as follows (Figure 2):

(1) Inflammation. Increased uric acid levels in the blood promoted the expression of interleukin-1 $\beta$ (IL- $1 \beta$ ), interleukin-6 (IL-6), tumor necrosis factor- $\alpha$ (TNF$\alpha)$ [19], and CRP production [20]. In animal studies, the activation of inflammation induced by UA decreases insulin sensitivity in mice [21], and infusion of UA into mice can increase TNF- $\alpha$ levels and activate the classical inflammatory pathway [22]. In human studies, serum UA was positively associated with TNF- $\alpha$, interleukin- 6 and C-reactive protein in healthy people [23].

(2) Oxidative Stress. Excessive uric acid will lead to an increase in reactive oxygen species (ROS) production, which leads to inflammation and dysfunction in the vessel [24]. UA is a powerful antioxidant that can remove superoxide and hydroxyl radicals in plasma, and UA has prooxidant effects in vascular tissue by increasing ROS production, such as $\mathrm{H}_{2} \mathrm{O}_{2}$ [24]. UA-mediated oxidative stress-induced lipid peroxidation, DNA damage, and activation of inflammatory factors finally lead to cellular damage [24]. Oxidative stress also can affect the expression of insulin gene, causing a decrease in insulin secretion [25].
(3) Endothelial Dysfunction. Endothelial dysfunction is characterized by deficiencies in the synthesis and/or bioavailability of endothelium-derived NO [26]. In addition, UA reduces endothelial NO bioavailability in humans [27]. Uric acid inhibits proliferation and migration of endothelial cells and NO secretion [20]. $\mathrm{UA}$ can react with $\mathrm{NO}$ to form 6-aminouracil, UAdependent ROS reacts with NO to form peroxynitrite, and UA can hold back L-arginine uptake and stimulate L-arginine degradation [6]. As a result of the effects of hyperglycemia and neurohormonal activation, UA levels are independently associated with endothelial dysfunction in animals and humans, thereby promoting hypertension [28].

(4) Inhibiting Insulin Pathway. UA directly inhibits the trigger of insulin signaling pathway by an ectonucleotide pyrophosphatase/phosphodiesterase 1 (ENPP1) recruitment at the receptor level [29].

All factors interference with glucose homeostasis and insulin sensitivity promotes the development of diabetes [30-32].

2.2. Uric Acid and Diabetic Chronic Complications. The aforementioned changes to diabetes are also directly related to the metabolic disorder: desulfation of glycosaminoglycans (GAGs) and formation of advanced glycation end products (AGE) and receptors (RAGE) [33]. It is widely believed that polyol bypass, protein kinase $\mathrm{C}$, hexosamine activation, advanced glycosylation products (AGEs), increased hyperglycemia-induced mitochondria production of reactive oxygen species (ROS), inflammation, and endothelial dysfunction are the common pathogenic characteristics of chronic complications of diabetes mellitus [10,33-39], which mainly include macroangiopathy, microangiopathy, and neuropathy. Two other mechanisms are associated with chronic complications as follows (Figure 2):

(1) Activation of RAAS. Uric acid can lead to the activation of the renin-angiotensin-aldosterone System (RAAS), through increasing the production of juxtaglomerular renin [40]. UA-induced ROS stimulated the increase of plasma angiotensin II which induced aldosterone release, leading to activation of RAAS [24, 41]. RAAS activation induced afferent renal arteriolopathy and tubulointerstitial fibrosis in rodent models [42]. In diabetes, RAAS activation causes a range of pathological changes including vascular dysfunction, high intraglomerular pressure, inflammation, and so on, leading to cardiovascular and renal complications [43].

(2) Thrombus. Uric acid seems to trigger platelet adhesion and aggregation, thus favoring vascular thrombosis [44].

\section{Epidemiology Studies}

3.1. Uric Acid and Diabetes. The relationship between uric acid and diabetes has gradually become a hot topic of 


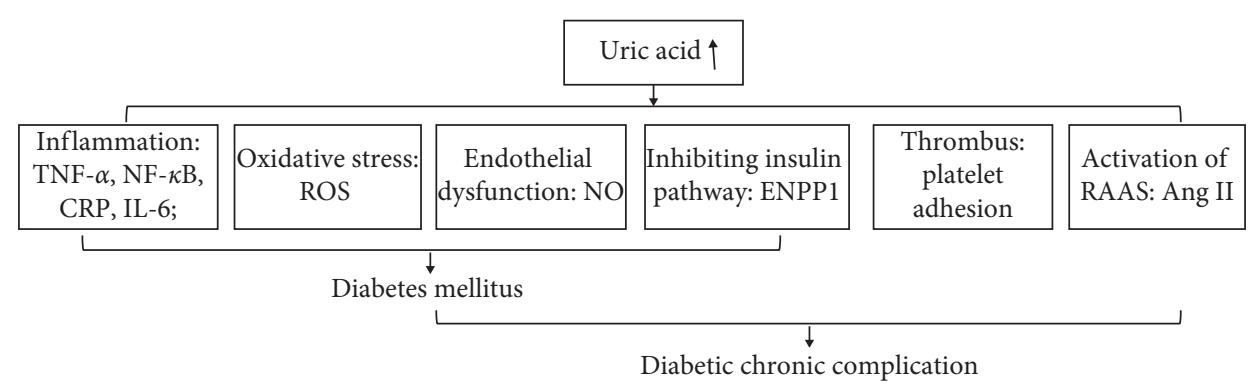

Figure 2: Metabolism of uric acid leading to diabetes mellitus and its chronic complication.

research, but controversy still exists. On the one hand, some study reported uric acid was not associated with diabetes. For example, Sluijs et al. [45] used a genetic score of 24 uric acid-related sites for Mendelian randomization studies, in the European prospective survey data-Cancer and Nutrition (EPIC) study, which was an interactive case-cohort study of vast number of subjects from eight European countries. In EPIC, after a mean of 10 years of follow-up, the results suggested that hyperuricemia was not salient associated with a higher risk of diabetes after adjusting for interference factors when their participant number was increased from 10,576 to 41,508 . Similarly, a large prospective cohort study was performed by $\mathrm{Li}$ [46] who followed up 4412 nondiabetic patients for 4.7 years to study urate changes in glucose metabolism. They found the uric acid concentration was not related to an increased risk of type 2 diabetes mellitus (T2DM).

On the opposite hand, more clinical trials demonstrated uric acid was significantly associated with diabetes. For example, Bombelli et al. [47, 48] randomly selected 3,200 northern Italian residents between the ages of 25 and 74 and found that increased uric acid resulted in an increased risk of impaired fasting glucose (IFG), and people with higher median UA levels may also develop metabolic syndrome and diabetes. In women, serum uric acid (SUA) levels in the normal range were associated with an increased risk of newonset diabetes compared with women with low-normal values [49]. Older adults with high levels of uric acid $(6.0 \mathrm{mg} /$ $\mathrm{dl}$ for men and $5.5 \mathrm{mg} / \mathrm{dl}$ for women) were more susceptible to metabolic syndrome and T2DM, especially in the 7584 years age group [50]. Serum UA was an important predictor of risk of metabolic syndrome, diabetes, and hypertension in adult males [51]. However, the relationship between blood UA and decreased insulin sensitivity in patients with type 1 diabetes mellitus is weaker than in healthy subjects [52].

Through reading a large number of literature and studies, we believe that uric acid is closely related to diabetes. Poor lipid metabolism in individuals with higher UA levels may lead to increased fasting and postprandial insulin levels, high-sensitivity C-reactive protein, hepatic insulin resistance index, and decreased glomerular filtration rate and skeletal muscle insulin sensitivity; high levels of SUA may impair liver insulin sensitivity and insulin clearance [53]. Perticone F [54] was documented when hypertensive NGT $\geq 155 \mathrm{mmHg}$, and UA is closely related to 1-h postload glucose during an oral glucose tolerance. We [55] analyzed the clinical characteristics and islet function index of 403 newly diagnosed patients with T2DM (mean age, $50.21 \pm 13.34$ years old; $62.5 \%$ male) and analyzed the SUA levels according to gender. Multivariate linear regression analysis showed that SUA had an independent effect on insulin secretion in female patients; the islet $\beta$-cell function of male was also affected by SUA, age, body mass index (BMI), and blood lipids; SUA correlated positively with insulin secretion and the insulin resistance index in male patients.

In terms of gestational diabetes, Leng [56] found that the SUA level is positively related with the risk of T2DM and prediabetes in the Tianjin region of China gestational diabetes mellitus (GDM) prevention planning data. In the group with GDM and impaired glucose tolerance (IGT), the mean SUA level was significantly increased in early pregnancy, and a UA level of $3.95 \mathrm{mg} / \mathrm{dl}$ could predict GDM with $60 \%$ specificity and $100 \%$ sensitivity [57].

\subsection{Uric Acid and Diabetic Chronic Complications}

3.2.1. Uric Acid and Diabetic Macrovascular Disease. Diabetic macroangiopathy refers to atherosclerosis of blood vessels such as the aorta, coronary artery, basilar artery, renal artery, and peripheral arteries, especially in the heart and cerebrovascular diseases, which is caused by dysfunction of endothelial cells, advanced glycation end product (AGEs/ RAGEs) system, the hexosamine pathway, inflammation, oxidant stress, protein kinase (PKC), and polyol [34-37]. Some clinical studies have shown a positive correlation between uric acid and diabetic macroangiopathy. Yan et al. [58] used Mendelian randomized analysis to determine whether there is a causal relationship between UA and diabetic macrovascular disease and found that the prevalence of diabetic macrovascular disease was significantly higher in the hyperuricemia group than in the healthy population, suggesting that UA and diabetic macrovascular disease are related. Indeed, the link between female-weighted genetic risk score (GRS) and diabetic macrovascular disease was greater than expected. Hyperuricemia was also observed to be associated with an increased incidence of atrial fibrillation in hospitalized patients with T2DM [59]. Hyperuricemia can increase the risk of sudden atrial fibrillation by approximately four-fold [60] and is associated with cardiovascular mortality [61]. Cardiovascular and cerebrovascular diseases are mainly caused by ischemia and hypoxia resulting from coronary atherosclerosis. Du et al. [62] performed a metaanalysis of patients with T2DM to determine whether SUA 
levels were associated with cerebral infarction and calculated the ratio of means (RoM) for SUA and the average cerebral infarction or average diabetes control ratio of individual studies and then compared it with the calculated $95 \%$ confidence intervals. The results showed that higher SUA levels might lead to cerebral infarction in patients with T2DM. Wang et al. [63] used the "Comprehensive Diabetes Prevention and Control Study (CRPCD)" data to explore the relationship between SUA and ischemic stroke in patients with T2DM in China. A total of 19,442 participants were enrolled in a cross-sectional study. The SUA level was significantly higher in patients over 60 years of age than in people under 60 years of age. Serum UA levels were independently and positively correlated with ischemic stroke in patients under 60 years of age, and it was characterized by U-type association in patients over 60 years of age. We speculated that the incidence of other established stroke risk factors such as hypertension, dyslipidemia, and chronic kidney disease increased with age would made it difficult to establish UA as an independent role in stroke.

Diabetic hyperglycemia causes metabolic abnormalities, which can affect systemic organs. Diabetic foot is caused by peripheral vascular disease, peripheral (motor, sensory, and autonomic) neuropathy, and excessive mechanical stress (repetitive external or minor trauma) in diabetic patients, leading to the destruction and deformity of the soft tissue and bone joint system of the foot [64]. The pathogenesis is partly the same as diabetic vascular and neuropathy complications [65]. Uric acid can be used as an independent risk factor to assess the development of diabetic foot [66].

3.2.2. Uric Acid and Diabetic Microangiopathy. Diabetic microangiopathy is a specific complication of diabetes. The typical changes comprise microcirculatory disorders and microvascular basement membrane thickening, which mainly lead to diabetic nephropathy (DN) [51] and diabetic retinopathy (DR) [67-70].

(1) Uric Acid and Diabetic Nephropathy. Diabetic nephropathy is a long-standing microvascular complication of diabetes and is the leading cause of end-stage renal disease in developed countries [10,71]. As an inflammatory factor, UA increases oxidative stress and promotes the activation of the renin-angiotensin-aldosterone system (RAAS) [21, 41]. Therefore, UA levels are associated with the occurrence and development of DN and are independent risk factors for early kidney disease [72, 73], which help to predict microalbuminuria progression [74]. Serum UA and microalbuminuria levels were significantly positively correlated with renal disease in patients with T2DM [75]. Patients with higher SUA levels have poorer renal function, independent of glycated hemoglobin (HbAlc) or the duration of diabetes [76]. In T2DM, there is an independent and significant positive association between higher blood UA and an increased risk of a reduced glomerular filtration rate (eGFR) [77]. Blood UA levels greater than $5.5 \mathrm{mg} / \mathrm{dl}$ can predict chronic kidney disease of stage 3 and above in T2DM [78]. The level of SUA that protects against progression of type 2 diabetic nephropathy (diabetic kidney disease (DKD)) is lower than the current normal value. The optimal cut-off value is $377.5 \mu \mathrm{mol} / \mathrm{l}(6.3 \mathrm{mg} / \mathrm{dl})$ for men and $309.0 \mu \mathrm{mol} / \mathrm{l}$ $(5.2 \mathrm{mg} / \mathrm{dl})$ for women [79]. In Chinese patients with T2DM, UA-related alleles such as SLC2A9 rs11722228 (solute carrier family 2 member 9), SLC2A9 rs3775948, and ABCG2 rs2231142 (ATP binding cassette subfamily G member 2) may affect susceptibility to DKD [80]. Contrast-enhanced ultrasound (CEUS) was used to show renal microvascular hyperperfusion, with a decreased glomerular filtration rate and reduced UA excretion in patients with DKD [81]. Xanthine oxidase $(\mathrm{XO})$ is a very important enzyme that is responsible for the conversion of sulfhydryl groups to UA. Elevation of UA by $1 \mu \mathrm{mol} / 1$ enhanced the probability of albuminuria by $1.5 \%$, and a rise in $\mathrm{XO}$ activity of $1 \mathrm{U} / \mathrm{l}$ also increased the probability of albuminuria by $1.5 \%$. In diabetes, both $\mathrm{XO}$ and uric acid are independently associated with albuminuria [82].

In patients with type 1 diabetes without complications, higher UA levels are associated with lower GFR, which is due to UA-mediated increased resistance in afferent renal arteriole promoting the renal microcirculation ischemia $[83,84]$.

In type 1 diabetes, kidney damage is more common in men whose SUA and creatinine concentrations and the albumin excretion rate are higher than those in female patients. Indeed, hyperglycemia adversely affects the activity of estrogen receptors (ER) and this may be gender-specific. The progression of renal disease in men with T1D is associated with a decline in free estradiol levels [85], and $17 \beta$ estradiol shows antioxidant, antiapoptotic, and anti-inflammatory properties [86]. The SUA level in boys but not girls with T1D was positively correlated with subclinical inflammation marker levels (CRP, IL-6, TNF- $\alpha$ ), renal function indicators (albumin excretion rate, cystatin-C level), and blood pressure; it was negatively correlated with anti-inflammatory IL-10 [87].

(2) Uric Acid and Diabetic Retinopathy. Diabetic retinopathy (DR) is a specific fundus lesion that is the main cause of blindness in patients with diabetes [88]. Based on the changes of haemodynamics or vascular geometry, vascular injury is considered to be the prime motivator for the initiation and progression of $\mathrm{DR}$, including pericytosis, platelet aggregation, thickening of basement membrane, and neuroglial damage [89]. The blood retinal barrier, as precondition to vision acuity, is vulnerable to injury during the progression of DR. This is a consequence of the interplay of AGE, hexosamine, polyol, inflammation, NO decline, oxidative stress, PKC, and RAS [38]. Uric acid is closely related to these pathological changes. Clinically, DR is classified into nonproliferative diabetic retinopathy (NPDR) (also known as simple type or background type) and proliferative diabetic retinopathy (PDR), according to whether or not retinal neovascularization occurs [90]. In Chinese patients with T2DM, reduction in urinary uric acid excretion (UUAE) is an independent risk factor for DR [91]. Elevated SUA levels are significantly associated with albuminuria and DR severity [92], but not with the retinal nerve fibre layer or 
macular thickness [93]. A study reported that increased SUA levels were associated with an increased severity of DR in Taiwan [94]. Kuwata [95] analyzed data from 1839 patients with T2DM in Japan by gender stratification and found that higher SUA levels were associated with an increased risk of DR in men, but not in women. The results showed sex hormones play an important role in the metabolism of uric acid, which deserved to discuss the specific mechanism further.

3.2.3. Uric Acid and Diabetic Peripheral Neuropathy. Diabetic neuropathy is one of the most common chronic complication of diabetes [96], characterized by damage to nerve glial cells, axons, and endothelial cells, and the morbidity from $30 \%$ to $50 \%$ in T2DM [97]. Diabetic peripheral neuropathy (DPN) is the main clinical manifestation of sensory and autonomic nerve symptoms, distal symmetry polyneuropathy, and motor neuropathy are the most common types of DPN [98]. The pathophysiology changes conclude polyol pathway, PKC activity, increased AGEs, oxidative stress (ROS), inflammation (IL-1 $\beta$, IL-6, $\mathrm{TNF} \alpha$, and $\mathrm{COX}-2$ ), microvascular alterations (endothelial dysfunction), nerve degeneration and regrowth (MMPs, Schwann cells and ECM), and the changes of the bloodnerve barrier [39, 99, 100]. Lin et al. [101] observed significant differences in the ratio of motor and sensory nerve amplitude and conduction velocity (CV) parameters between groups with different blood UA levels (both $P<0.05$ ). Blood UA levels were negatively correlated with the ratio of motor and sensory nerve amplitude and CV. Blood UA at $9 \mathrm{mg} / \mathrm{dl}$ and total cholesterol of $5.2 \mathrm{mmol} / \mathrm{l}$ were significantly associated with DPN in patients who had suffered from T2DM for more than 10 years. Yu et al. [102] performed a meta-analysis of 1388 patients with T2DM with peripheral neuropathy and in 4746 patients without peripheral neuropathy and showed that SUA levels were significantly elevated in patients with diabetes complicated with peripheral neuropathy and that increased hyperuricemia was related with increased risk of peripheral neuropathy.

\section{Conclusion}

Complex genetic and environmental factors contribute to causing diabetes, and chronic complications of diabetes may occur throughout the body. The pathogenesis of T2DM is complex, involving various interacting factors. Its increased incidence rate is a great concern worldwide. Hyperuricemia is closely related to the development of diabetes and its chronic complications. Many animal and human experiments have confirmed that UA mainly affects diabetes and its complications through inflammation, oxidative stress, endothelial function damage, and other effects. We call for further researches to explore the molecular mechanism, especially in the direct effect of uric acid on insulin secretion.

\section{Conflicts of Interest}

The authors declare that there are no conflicts of interest related with the publication of this paper.

\section{Authors' Contributions}

Qing Xiong and Jie Liu contributed equally to this work.

\section{Acknowledgments}

This study was supported by the National Natural Science Foundation of China (no. 81370872). The authors are indebted to the Department of Endocrinology, Zhongnan Hospital of Wuhan University, for providing suggestion during the preparation of the manuscript.

\section{References}

[1] S. A. Hannou, D. E. Haslam, N. M. McKeown, and M. A. Herman, "Fructose metabolism and metabolic disease," Journal of Clinical Investigation, vol. 128, no. 2, pp. 545-555, 2018.

[2] M. A. Lanaspa, C. Cicerchi, G. Garcia et al., "Counteracting roles of AMP deaminase and AMP kinase in the development of fatty liver," PLoS One, vol. 7, no. 11, Article ID e48801, 2012.

[3] M. A. Lanaspa, L. G. Sanchez-Lozada, Y.-J. Choi et al., "Uric acid induces hepatic steatosis by generation of mitochondrial oxidative stress," Journal of Biological Chemistry, vol. 287, no. 48, pp. 40732-40744, 2012.

[4] C. Cicerchi, N. Li, J. Kratzer et al., "Uric acid-dependent inhibition of AMP kinase induces hepatic glucose production in diabetes and starvation: evolutionary implications of the uricase loss in hominids," The FASEB Journal, vol. 28, no. 8, pp. 3339-3350, 2014.

[5] T. Ishimoto, M. A. Lanaspa, M. T. Le et al., "Opposing effects of fructokinase $\mathrm{C}$ and $\mathrm{A}$ isoforms on fructose-induced metabolic syndrome in mice," Proceedings of the National Academy of Sciences, vol. 109, no. 11, pp. 4320-4325, 2012.

[6] R. J. Johnson, T. Nakagawa, L. G. Sanchez-Lozada et al., "Sugar, uric acid, and the etiology of diabetes and obesity," Diabetes, vol. 62, no. 10, pp. 3307-3315, 2013.

[7] A. C. M. Gagliardi, M. H. Miname, and R. D. Santos, "Uric acid: a marker of increased cardiovascular risk," Atherosclerosis, vol. 202, no. 1, pp. 11-17, 2009.

[8] T. Du, X. Sun, H. Lu et al., "Associations of serum uric acid levels with cardiovascular health factors:Differences by sex, age and body mass index in Chinese participants," European Journal of Internal Medicine, vol. 25, no. 4, pp. 388-393, 2014.

[9] R. J. Johnson, T. Merriman, and M. A. Lanaspa, "Causal or noncausal relationship of uric acid with diabetes: table 1," Diabetes, vol. 64, no. 8, pp. 2720-2722, 2015.

[10] Y. Lytvyn, B. A. Perkins, and D. Z. I. Cherney, "Uric acid as a biomarker and a therapeutic target in diabetes," Canadian Journal of Diabetes, vol. 39, no. 3, pp. 239-246, 2015.

[11] E. B. Sochett, D. Z. I. Cherney, J. R. Curtis, M. G. Dekker, J. W. Scholey, and J. A. Miller, "Impact of renin angiotensin system modulation on the hyperfiltration state in type 1 diabetes," Journal of the American Society of Nephrology, vol. 17, no. 6, pp. 1703-1709, 2006.

[12] I. A. Bobulescu and O. W. Moe, "Renal transport of uric acid: evolving concepts and uncertainties," Advances in Chronic Kidney Disease, vol. 19, no. 6, pp. 358-371, 2012.

[13] J. Maesaka and S. Fishbane, "Regulation of renal urate excretion: a critical review," American Journal of Kidney Diseases, vol. 32, no. 6, pp. 917-933, 1998. 
[14] L. B. Sorensen and D. J. Levinson, "Origin and extrarenal elimination of uric acid in man," Nephron, vol. 14, no. 1, pp. 7-20, 1975.

[15] T. J. Gibson, "Hypertension, its treatment, hyperuricaemia and gout," Current Opinion in Rheumatology, vol. 25, no. 2, pp. 217-222, 2013.

[16] S. Bonakdaran and B. Kharaqani, "Association of serum uric acid and metabolic syndrome in type 2 diabetes," Current Diabetes Reviews, vol. 10, no. 2, pp. 113-117, 2014.

[17] Y.-1. Li, H. Xie, H. Musha et al., "The risk factor Analysis for type 2 diabetes mellitus patients with nonalcoholic fatty liver disease and positive correlation with serum uric acid," Cell Biochemistry and Biophysics, vol. 72, no. 3, pp. 643-647, 2015.

[18] F. Viazzi, G. Leoncini, M. Vercelli, G. Deferrari, and R. Pontremoli, "Serum uric acid levels predict new-onset type 2 diabetes in hospitalized patients with primary hypertension: the MAGIC study," Diabetes Care, vol. 34, no. 1, pp. 126-128, 2011.

[19] R. J. Johnson, D.-H. Kang, D. Feig et al., "Is there a pathogenetic role for uric acid in hypertension and cardiovascular and renal disease?," Hypertension, vol. 41, no. 6, pp. 1183-1190, 2003.

[20] D.-H. Kang, S.-K. Park, I.-K. Lee, and R. J. Johnson, "Uric acid-induced C-reactive protein expression: implication on cell proliferation and nitric oxide production of human vascular cells," Journal of the American Society of Nephrology, vol. 16, no. 12, pp. 3553-3562, 2005.

[21] K. Chaudhary, K. Malhotra, J. Sowers, and A. Aroor, "Uric acid-key ingredient in the recipe for cardiorenal metabolic syndrome," Cardiorenal Medicine, vol. 3, no. 3, pp. 208-220, 2013.

[22] D. M. Maahs, L. Caramori, D. Z. I. Cherney et al., "Uric acid lowering to prevent kidney function loss in diabetes: the preventing early renal function loss (PERL) allopurinol study," Current Diabetes Reports, vol. 13, no. 4, pp. 550-559, 2013.

[23] B. Kirilmaz, F. Asgun, E. Alioglu et al., "High inflammatory activity related to the number of metabolic syndrome components," The Journal of Clinical Hypertension, vol. 12, no. 2, pp. 136-144, 2010.

[24] M. A. Yu, L. G. Sanchez-Lozada, R. J. Johnson et al., “Oxidative stress with an activation of the renin-angiotensin system in human vascular endothelial cells as a novel mechanism of uric acid-induced endothelial dysfunction," Journal of Hypertension, vol. 28, no. 6, pp. 1234-1242, 2010.

[25] T. Matsuoka, Y. Kajimoto, H. Watada et al., "Glycationdependent, reactive oxygen species-mediated suppression of the insulin gene promoter activity in HIT cells," Journal of Clinical Investigation, vol. 99, no. 1, pp. 144-150, 1997.

[26] W. A. Hsueh, C. J. Lyon, and M. J. Quiñones, "Insulin resistance and the endothelium," The American Journal of Medicine, vol. 117, no. 2, pp. 109-117, 2004.

[27] C. Zoccali, R. Maio, F. Mallamaci, G. Sesti, and F. Perticone, "Uric acid and endothelial dysfunction in essential hypertension," Journal of the American Society of Nephrology, vol. 17, no. 5, pp. 1466-1471, 2006.

[28] D. Erdogan, H. Gullu, M. Caliskan et al., "Relationship of serum uric acid to measures of endothelial function and atherosclerosis in healthy adults," International Journal of Clinical Practice, vol. 59, no. 11, pp. 1276-1282, 2005.

[29] E. J. Tassone, A. Cimellaro, M. Perticone et al., "Uric acid impairs insulin signaling by promoting Enpp1 binding to insulin receptor in human umbilical vein endothelial cells," Frontiers in Endocrinology, vol. 9, no. 98, 2018.

[30] F. Perticone, R. Maio, A. Sciacqua et al., "Endothelial dysfunction and C-reactive protein are risk factors for diabetes in essential hypertension," Diabetes, vol. 57, no. 1, pp. 167171, 2008.

[31] W. Baldwin, S. McRae, G. Marek et al., "Hyperuricemia as a mediator of the proinflammatory endocrine imbalance in the adipose tissue in a murine model of the metabolic syndrome," Diabetes, vol. 60, no. 4, pp. 1258-1269, 2011.

[32] R. Spiga, M. A. Marini, E. Mancuso et al., "Uric acid is associated with inflammatory biomarkers and induces inflammation via activating the NF- $\kappa \mathrm{B}$ signaling pathway in HepG2 cells," Arteriosclerosis, Thrombosis, and Vascular Biology, vol. 37, no. 6, pp. 1241-1249, 2017.

[33] S. Coccheri, "Approaches to prevention of cardiovascular complications and events in diabetes mellitus," Drugs, vol. 67, no. 7, pp. 997-1026, 2007.

[34] D. Laight, M. J. Carrier, and E. E. Anggard, "Antioxidants, diabetes and endothelial dysfunction," Cardiovascular Research, vol. 47, no. 3, pp. 457-464, 2000.

[35] D. W. Laight, M. J. Carrier, and E. E. Änggård, "Endothelial cell dysfunction and the pathogenesis of diabetic macroangiopathy," Diabetes/Metabolism Research and Reviews, vol. 15, no. 4, pp. 274-282, 1999.

[36] R. Madonna, D. Pieragostino, C. R. Balistreri et al., "Diabetic macroangiopathy: pathogenetic insights and novel therapeutic approaches with focus on high glucose-mediated vascular damage," Vascular Pharmacology, vol. 107, pp. 2734, 2018.

[37] R. Madonna and R. De Caterina, "Cellular and molecular mechanisms of vascular injury in diabetes-Part I: pathways of vascular disease in diabetes," Vascular Pharmacology, vol. 54, no. 3-6, pp. 68-74, 2011.

[38] N. Mahajan, P. Arora, and R. Sandhir, "Perturbed biochemical pathways and associated oxidative stress lead to vascular dysfunctions in diabetic retinopathy," Oxidative Medicine and Cellular Longevity, vol. 2019, Article ID 8458472, 16 pages, 2019.

[39] G. J. Bönhof, C. Herder, A. Strom, N. Papanas, M. Roden, and D. Ziegler, "Emerging biomarkers, tools, and treatments for diabetic polyneuropathy," Endocrine Reviews, vol. 40, no. 1, pp. 153-192, 2019.

[40] M. Mazzali, J. Hughes, Y.-G. Kim et al., "Elevated uric acid increases blood pressure in the rat by a novel crystal-independent mechanism," Hypertension, vol. 38, no. 5, pp. 1101-1106, 2001.

[41] V. Filiopoulos, D. Hadjiyannakos, and D. Vlassopoulos, "New insights into uric acid effects on the progression and prognosis of chronic kidney disease," Renal Failure, vol. 34, no. 4, pp. 510-520, 2012.

[42] M. Mazzali, J. Kanellis, L. Han et al., "Hyperuricemia induces a primary renal arteriolopathy in rats by a blood pressureindependent mechanism," American Journal of PhysiologyRenal Physiology, vol. 282, no. 6, pp. F991-F997, 2002.

[43] R. Zatz, T. W. Meyer, H. G. Rennke, and B. M. Brenner, "Predominance of hemodynamic rather than metabolic factors in the pathogenesis of diabetic glomerulopathy," Proceedings of the National Academy of Sciences, vol. 82, no. 17, pp. 5963-5967, 1985.

[44] M. H. Ginsberg, F. Kozin, M. O’Malley, and D. J. McCarty, "Release of platelet constituents by monosodium urate crystals," Journal of Clinical Investigation, vol. 60, no. 5, pp. 999-1007, 1977. 
[45] I. Sluijs, M. V. Holmes, Y. T. van der Schouw et al., "A mendelian randomization study of circulating uric acid and type 2 diabetes," Diabetes, vol. 64, no. 8, pp. 3028-3036, 2015.

[46] X. Li, X. Meng, X. C. Gao et al., "Elevated serum xanthine oxidase activity is associated with the development of type 2 diabetes: a prospective cohort study," Diabetes Care, vol. 41, no. 4, pp. 884-890, 2018.

[47] M. Bombelli, F. Quarti-Trevano, M. Tadic et al., "Uric acid and risk of new-onset metabolic syndrome, impaired fasting glucose and diabetes mellitus in a general Italian population," Journal of Hypertension, vol. 36, no. 7, pp. 14921498, 2018.

[48] T. Anothaisintawee, D. Lertrattananon, S. Thamakaison et al., "Direct and indirect effects of serum uric acid on blood sugar levels in patients with prediabetes: a mediation analysis," Journal of Diabetes Research, vol. 2017, Article ID 6830671, 6 pages, 2017.

[49] M. Shani, S. Vinker, D. Dinour et al., "High normal uric acid levels are associated with an increased risk of diabetes in lean, normoglycemic healthy women," The Journal of Clinical Endocrinology and Metabolism, vol. 101, no. 10, pp. 37723778, 2016.

[50] J.-B. Chang, Y.-L. Chen, Y.-J. Hung et al., "The role of uric acid for predicting future metabolic syndrome and type 2 diabetes in older people," The Journal of Nutrition, Health and Aging, vol. 21, no. 3, pp. 329-335, 2017.

[51] Y.-Y. Chen, T.-W. Kao, H.-F. Yang et al., “The association of uric acid with the risk of metabolic syndrome, arterial hypertension or diabetes in young subjects- an observational study," Clinica Chimica Acta, vol. 478, pp. 68-73, 2018.

[52] P. Bjornstad, J. K. Snell-Bergeon, K. McFann et al., "Serum uric acid and insulin sensitivity in adolescents and adults with and without type 1 diabetes," Journal of Diabetes and Its Complications, vol. 28, no. 3, pp. 298-304, 2014.

[53] T. V. Fiorentino, F. Sesti, E. Succurro et al., "Higher serum levels of uric acid are associated with a reduced insulin clearance in non-diabetic individuals," Acta Diabetologica, vol. 55, no. 8, pp. 835-842, 2018.

[54] F. Perticone, A. Sciacqua, M. Perticone et al., "Serum uric acid and 1-h postload glucose in essential hypertension," Diabetes Care, vol. 35, no. 1, pp. 153-157, 2012.

[55] Y. Hu, J. Liu, H. Li et al., "The association between elevated serum uric acid levels and islet $\beta$-cell function indexes in newly diagnosed type 2 diabetes mellitus: a cross-sectional study," PeerJ, vol. 6, p. e4515, 2018.

[56] J. Leng, L. Wang, J. Wang et al., "Uric acid and diabetes risk among Chinese women with a history of gestational diabetes mellitus," Diabetes Research and Clinical Practice, vol. 134, pp. 72-79, 2017.

[57] S. S. Aker, T. Yuce, E. Kalafat et al., "Association of first trimester serum uric acid levels gestational diabetes mellitus development," Journal of Turkish Society of Obstetric and Gynecology, vol. 13, no. 2, pp. 71-74, 2016.

[58] D. D. Yan, J. Wang, F. Jiang et al., "A causal relationship between uric acid and diabetic macrovascular disease in Chinese type 2 diabetes patients: a Mendelian randomization analysis," International Journal of Cardiology, vol. 214, pp. 194-199, 2016.

[59] A. Mantovani, R. Rigolon, I. Pichiri et al., "Hyperuricemia is associated with an increased prevalence of atrial fibrillation in hospitalized patients with type 2 diabetes," Journal of Endocrinological Investigation, vol. 39, no. 2, pp. 159-167, 2016.
[60] A. Mantovani, R. Rigolon, A. Civettini et al., "Hyperuricemia is associated with an increased prevalence of paroxysmal atrial fibrillation in patients with type 2 diabetes referred for clinically indicated 24-h Holter monitoring," Journal of Endocrinological Investigation, vol. 41, no. 2, pp. 223-231, 2018.

[61] A. I. Ilundain-González, J. A. Gimeno-Orna, D. Sáenz-Abad, J. Pons-Dolset, J. Cebollada-del Hoyo, and M. d. C. LahozaPérez, "Influencia de los niveles de ácido úrico sobre el riesgo de mortalidad cardiovascular a largo plazo en pacientes con diabetes de tipo 2," Endocrinología, Diabetes Y Nutrición, vol. 65, no. 6, pp. 335-341, 2018.

[62] L. Du, J. Ma, and X. Zhang, "Higher serum uric acid may contribute to cerebral infarction in patients with type 2 diabetes mellitus: a meta-analysis," Journal of Molecular Neuroscience, vol. 61, no. 1, pp. 25-31, 2017.

[63] L. Wang, W. Hu, D. D. Miao et al., "Relationship between serum uric acid and ischemic stroke in a large type 2 diabetes population in China: a cross-sectional study," Journal of the Neurological Sciences, vol. 376, pp. 176-180, 2017.

[64] D. G. Armstrong, A. J. M. Boulton, and S. A. Bus, "Diabetic foot ulcers and their recurrence," New England Journal of Medicine, vol. 376, no. 24, pp. 2367-2375, 2017.

[65] M. Volmer-Thole and R. Lobmann, "Neuropathy and diabetic foot syndrome," International Journal of Molecular Sciences, vol. 17, no. 6, 2016.

[66] X. Ye, Y. Cao, F. Gao et al., "Elevated serum uric acid levels are independent risk factors for diabetic foot ulcer in female Chinese patients with type 2 diabetes," Journal of Diabetes, vol. 6, no. 1, pp. 42-47, 2014.

[67] A. J. Jaap and J. E. Tooke, "Pathophysiology of microvascular disease in non-insulin-dependent diabetes," Clinical Science, vol. 89, no. 1, pp. 3-12, 1995.

[68] K. F. Hanssen, "Blood glucose control and microvascular and macrovascular complications in diabetes," Diabetes, vol. 46, no. 2, pp. S101-S103, 1997.

[69] F. Persson and P. Rossing, "Diagnosis of diabetic kidney disease: state of the art and future perspective," Kidney International Supplements, vol. 8, no. 1, pp. 2-7, 2018.

[70] F. Semeraro, F. Morescalchi, A. Cancarini et al., "Diabetic retinopathy, a vascular and inflammatory disease: therapeutic implications," Diabetes and Metabolism, pii: S12623636(19)30062-X, 2019.

[71] Y.-H. Chang, C.-C. Lei, K.-C. Lin, D.-M. Chang, C.-H. Hsieh, and Y.-J. Lee, "Serum uric acid level as an indicator for CKD regression and progression in patients with type 2 diabetes mellitus-a 4.6-year cohort study," Diabetes/Metabolism Research and Reviews, vol. 32, no. 6, pp. 557-564, 2016.

[72] S. De Cosmo, F. Viazzi, A. Pacilli et al., "Serum uric acid and risk of CKD in type 2 diabetes," Clinical Journal of the American Society of Nephrology, vol. 10, no. 11, pp. 19211929, 2015.

[73] G. X. Li, X. H. Jiao, and X. B. Cheng, "Correlations between blood uric acid and the incidence and progression of type 2 diabetes nephropathy," European Review for Medical and Pharmacological Sciences, vol. 22, no. 2, pp. 506-511, 2018.

[74] Y. Hayashino, S. Okamura, S. Tsujii, and H. Ishii, "Association of serum uric acid levels with the risk of development or progression of albuminuria among Japanese patients with type 2 diabetes: a prospective cohort study [Diabetes Distress and Care Registry at Tenri (DDCRT 10)]," Acta Diabetologica, vol. 53, no. 4, pp. 599-607, 2016.

[75] H. Latif, A. Iqbal, R. Rathore et al., "Correlation between serum uric acid level and microalbuminuria in type-2 
diabetic nephropathy," Pakistan Journal of Medical Sciences, vol. 33, no. 6, pp. 1371-1375, 2017.

[76] M. H. Pizarro, D. C. Santos, B. S. V. Barros et al., "Serum uric acid and renal function in patients with type 1 diabetes: a nationwide study in Brazil," Diabetology and Metabolic Syndrome, vol. 10, 2018.

[77] J. Wang, Y. K. Yu, X. W. Li et al., "Serum uric acid levels and decreased estimated glomerular filtration rate in patients with type 2 diabetes: a cohort study and meta-analysis," Diabetes/Metabolism Research and Reviews, vol. 34, no. 7, 2018.

[78] W. J. Kim, S. S. Kim, M. J. Bae et al., "High-normal serum uric acid predicts the development of chronic kidney disease in patients with type 2 diabetes mellitus and preserved kidney function," Journal of Diabetes and Its Complications, vol. 28, no. 2, pp. 130-134, 2014.

[79] V. Bartáková, K. Kuricová, L. Pácal et al., "Hyperuricemia contributes to the faster progression of diabetic kidney disease in type 2 diabetes mellitus," Journal of Diabetes and Its Complications, vol. 30, no. 7, pp. 1300-1307, 2016.

[80] D. D. Yan, J. Wang, F. Jiang et al., "Association between serum uric acid related genetic loci and diabetic kidney disease in the Chinese type 2 diabetes patients," Journal of Diabetes and Its Complications, vol. 30, no. 5, pp. 798-802, 2016.

[81] L. Wang, J. F. Cheng, L. P. Sun et al., "Use of contrastenhanced ultrasound to study relationship between serum uric acid and renal microvascular perfusion in diabetic kidney disease," BioMed Research International, vol. 2015, Article ID 732317, 10 pages, 2015.

[82] A. Klisic, G. Kocic, N. Kavaric, M. Jovanovic, V. Stanisic, and A. Ninic, "Xanthine oxidase and uric acid as independent predictors of albuminuria in patients with diabetes mellitus type 2," Clinical and Experimental Medicine, vol. 18, no. 2, pp. 283-290, 2018.

[83] Y. Lytvyn, M. Škrtić, G. K. Yang, P. M. Yip, B. A. Perkins, and D. Z. I. Cherney, "Glycosuria-mediated urinary uric acid excretion in patients with uncomplicated type 1 diabetes mellitus," American Journal of Physiology-Renal Physiology, vol. 308, no. 2, pp. F77-F83, 2015.

[84] Y. Lytvyn, M. Skrtić, G. K. Yang et al., "Plasma uric acid effects on glomerular haemodynamic profile of patients with uncomplicated Type 1 diabetes mellitus," Diabetic Medicine, vol. 33, no. 8, pp. 1102-1111, 2016.

[85] C. Maric, C. Forsblom, L. Thorn, J. Wadén, and P.-H. Groop, "Association between testosterone, estradiol and sex hormone binding globulin levels in men with type 1 diabetes with nephropathy," Steroids, vol. 75, no. 11, pp. 772-778, 2010.

[86] A. A. Miller, G. R. Drummond, A. E. Mast, H. H. H. W. Schmidt, and C. G. Sobey, "Effect of gender on NADPH-oxidase activity, expression, and function in the cerebral circulation," Stroke, vol. 38, no. 7, pp. 2142-2149, 2007.

[87] B. Slominski, M. Skrzypkowska, M. Ryba-Stanislawowska et al., "Sex-related association of serum uric acid with inflammation, kidney function and blood pressure in type 1 diabetic patients," Pediatric Diabetes, vol. 19, no. 5, pp. 1014-1019, 2018.

[88] S. R. Flaxman, R. R. A. Bourne, S. Resnikoff et al., "Global causes of blindness and distance vision impairment 19902020: a systematic review and meta-analysis," The Lancet Global Health, vol. 5, no. 12, pp. e1221-e1234, 2017.

[89] E. Fletcher, J. Phipps, M. Ward, T. Puthussery, and J. Wilkinson-Berka, "Neuronal and glial cell abnormality as predictors of progression of diabetic retinopathy," Current Pharmaceutical Design, vol. 13, no. 26, pp. 2699-2712, 2007.

[90] A. R. Santiago, R. Boia, I. D. Aires et al., "Sweet stress: coping with vascular dysfunction in diabetic retinopathy," Frontiers in Physiology, vol. 9, p. 820, 2018.

[91] L.-X. Li, J.-X. Lu, H.-P. Shuai et al., "Decreased urine uric acid excretion is associated with diabetic retinopathy but not with lower limb atherosclerosis in hospitalized patients with type 2 diabetes," Atherosclerosis, vol. 242, no. 1, pp. 13-18, 2015.

[92] C. C. Liang, P. C. Lin, M. Y. Lee et al., "Association of serum uric acid concentration with diabetic retinopathy and albuminuria in Taiwanese patients with type 2 diabetes mellitus," International Journal of Molecular Sciences, vol. 17, no. 8, 2016.

[93] M. N. Vinuthinee-Naidu, E. Zunaina, A. Azreen-Redzal et al., "Correlation of retinal nerve fibre layer and macular thickness with serum uric acid among type 2 diabetes mellitus," BMC Ophthalmology, vol. 17, 2017.

[94] J.-J. Lee, I.-H. Yang, H.-K. Kuo et al., "Serum uric acid concentration is associated with worsening in severity of diabetic retinopathy among type 2 diabetic patients in Taiwan-A 3-year prospective study," Diabetes Research and Clinical Practice, vol. 106, no. 2, pp. 366-372, 2014.

[95] H. Kuwata, S. Okamura, Y. Hayashino et al., "Serum uric acid levels are associated with increased risk of newly developed diabetic retinopathy among Japanese male patients with type 2 diabetes: a prospective cohort study (diabetes distress and care registry at Tenri [DDCRT 13])," Diabetes/Metabolism Research and Reviews, vol. 33, no. 7, p. e2905, 2017.

[96] P. J. Dyck, K. M. Kratz, J. L. Karnes et al., "The prevalence by staged severity of various types of diabetic neuropathy, retinopathy, and nephropathy in a population-based cohort: the Rochester Diabetic Neuropathy Study," Neurology, vol. 43, no. 4, p. 817, 1993.

[97] A. Peltier, S. A. Goutman, and B. C. Callaghan, "Painful diabetic neuropathy," BMJ, vol. 348, no. 1, p. g1799, 2014.

[98] R. Pop-Busui, A. J. M. Boulton, E. L. Feldman et al., "Diabetic neuropathy: a position statement by the American diabetes association," Diabetes Care, vol. 40, no. 1, pp. 136-154, 2017.

[99] P. Shillo, G. Sloan, M. Greig et al., "Painful and painless diabetic neuropathies: what is the difference?," Current Diabetes Reports, vol. 19, no. 6, p. 32, 2019.

[100] M. Richner, N. Ferreira, A. Dudele et al., "Functional and structural changes of the blood-nerve-barrier in diabetic neuropathy," Frontiers in Neuroscience, vol. 12, p. 1038, 2018.

[101] X. Lin, L. Xu, D. Zhao, Z. Luo, and S. Pan, "Correlation between serum uric acid and diabetic peripheral neuropathy in T2DM patients," Journal of the Neurological Sciences, vol. 385, pp. 78-82, 2018.

[102] S. Yu, Y. Chen, X. Hou et al., "Serum uric acid levels and diabetic peripheral neuropathy in type 2 diabetes: a systematic review and meta-analysis," Molecular Neurobiology, vol. 53, no. 2, pp. 1045-1051, 2016. 


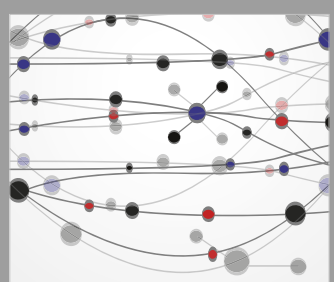

The Scientific World Journal
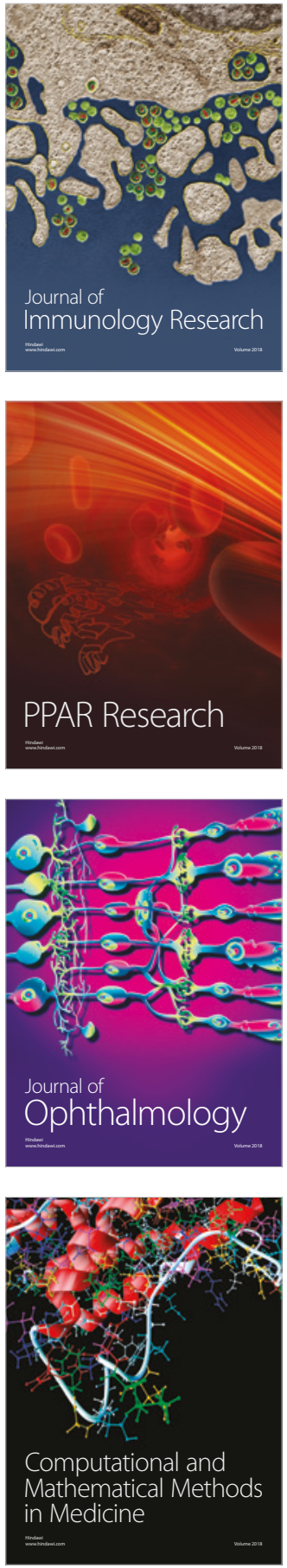

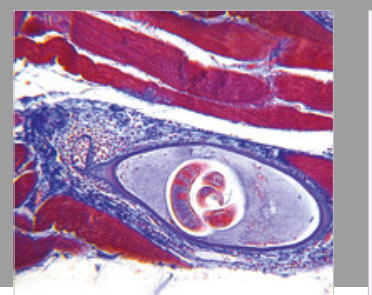

Gastroenterology Research and Practice

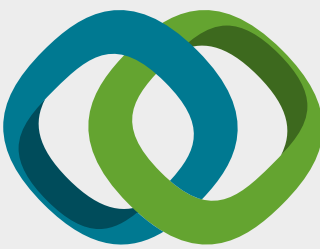

\section{Hindawi}

Submit your manuscripts at

www.hindawi.com
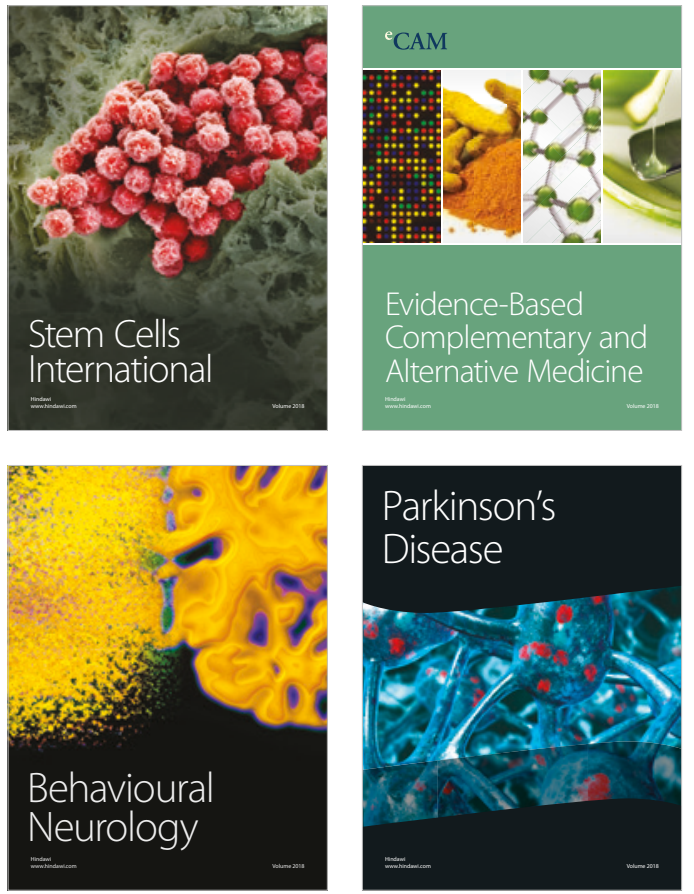

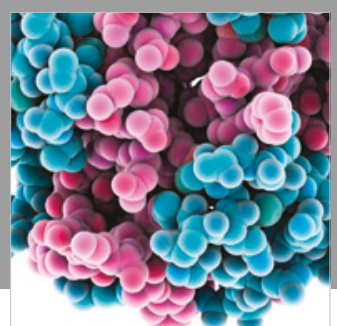

ournal of

Diabetes Research

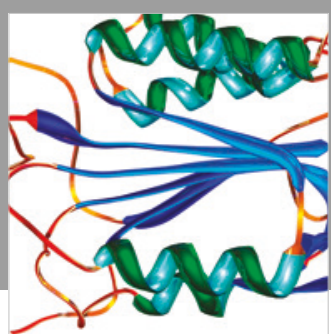

Disease Markers
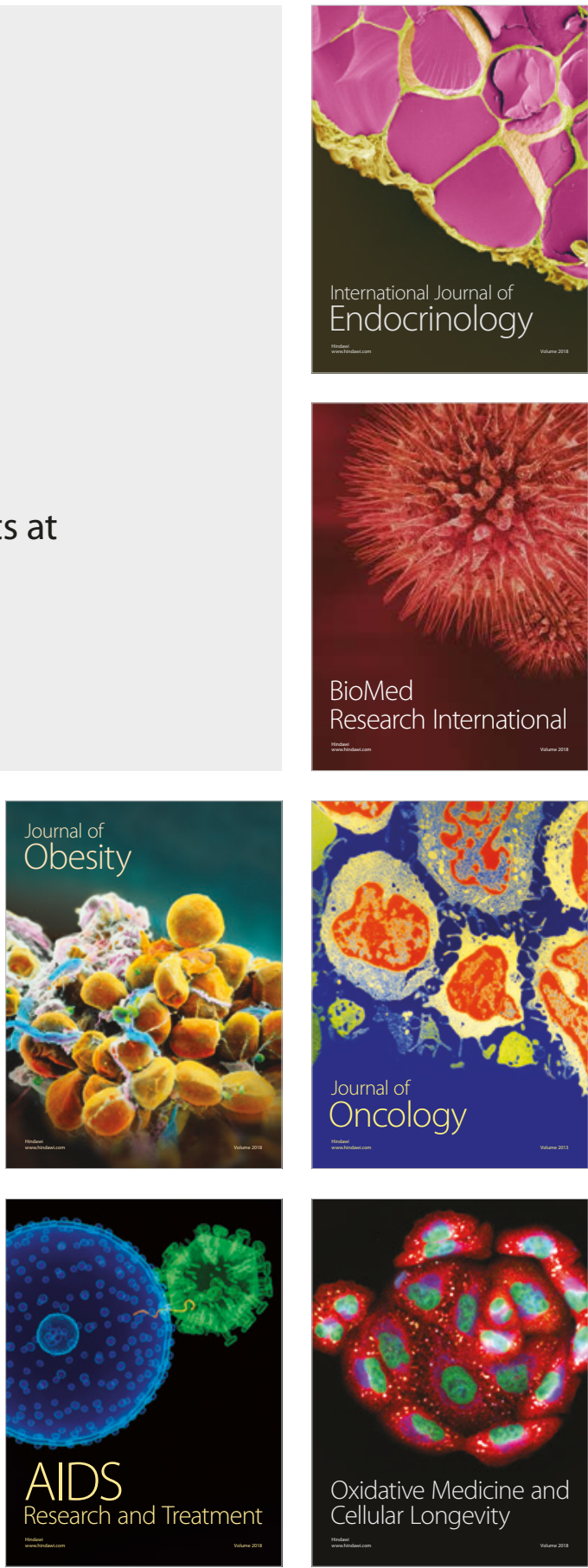